\title{
Correction to: Inconsistent Responses to Notifications of Suspected Plagiarism in Finnish Higher Education
}

\section{Erja Moore ${ }^{1}$ i}

Published online: 6 March 2021

(c) Springer Nature B.V. 2021

\section{Correction to: Journal of Academic Ethics (2020) 18:207-222 https://doi.org/10.1007/s10805-020-09366-1}

The original version of this article unfortunately contained a mistake. Out of an abundance of caution, we have removed Table 4 of Appendix 1 and replaced it with an anonymized table. Here is the corrected Table 4 of Appendix 1:

\section{Appendix 1}

Table 4 Notifications of suspected plagiarism in Master's theses (2018) and the content of rectors' decisions (2018-2019)

\begin{tabular}{|c|c|c|c|}
\hline & Year & Dec & ctor of the university \\
\hline 1 & 2003 & $\mathrm{XY}$ & $\begin{array}{l}\text { Decision published with appendices. Thesis marked with plagiarism statement. } \\
\text { (TENK) }\end{array}$ \\
\hline 2 & 2017 & $\mathrm{XY}$ & Re-evaluated, grade lowered. Thesis marked with plagiarism statement \\
\hline 3 & 2017 & $\mathrm{XY}$ & $\begin{array}{l}\text { Revoke of degree applied from Higher Administrative Court. Thesis marked } \\
\text { with plagiarism statement. (TENK) }\end{array}$ \\
\hline 4 & 2017 & $\mathrm{XY}$ & Student did not accept the preliminary inquiry. Investigation proper started \\
\hline 5 & 2017 & $\mathrm{XY}$ & $\begin{array}{l}\text { Re-evaluated, grade lowered. Thesis marked with plagiarism statement. Thesis } \\
\text { removed from Theseus. (TENK) }\end{array}$ \\
\hline 6 & 2018 & $\mathrm{XY}$ & $\begin{array}{l}\text { Re-evaluated, grade lowered. Thesis marked with plagiarism statement. } \\
\text { (TENK) }\end{array}$ \\
\hline 7 & 2015 & $\mathrm{XY}$ & $\begin{array}{l}\text { Should not have been accepted in the first place. Thesis marked with plagiarism } \\
\text { statement. (TENK) }\end{array}$ \\
\hline 8 & 2016 & $X Y$ & $\begin{array}{l}\text { Re-evaluated, grade lowered. Thesis marked with plagiarism statement. Thesis } \\
\text { removed from Theseus }\end{array}$ \\
\hline
\end{tabular}

The original article can be found online at https://doi.org/10.1007/s10805-020-09366-1.

Erja Moore

erja.moore@pkarjala.net

1 Joensuu, Finland 
Table 4 (continued)

\begin{tabular}{|c|c|c|c|}
\hline & Year & Deci & ector of the university \\
\hline$\overline{9}$ & 2015 & $\mathrm{XY}$ & $\begin{array}{l}\text { According to the decision the thesis will be marked with plagiarism statement } \\
\text { which has not been done by } 9.9 .2019\end{array}$ \\
\hline 10 & 2017 & $\mathrm{XY}$ & $\begin{array}{l}\text { Preliminary inquiry report attached to decision. Re-evaluated. Thesis marked } \\
\text { with plagiarism statement }\end{array}$ \\
\hline 11 & 2017 & $\mathrm{Z}$ & $\begin{array}{l}\text { No violation found. Due to carelessness thesis re-evaluated, degraded. Grade } \\
\text { was } X \text {, is now } X \text {. Notification inappropriate. (TENK) }\end{array}$ \\
\hline 12 & 2017 & $\mathrm{X}$ & $\begin{array}{l}\text { Re-evaluated. Thesis marked with plagiarism statement (an extra page after } \\
\text { cover page) }\end{array}$ \\
\hline 13 & 2012 & $\mathrm{~V}$ & $\begin{array}{l}\text { "The thesis has not in all respects fulfilled good scientific practice." Removed } \\
\text { from Theseus }\end{array}$ \\
\hline 14 & 2018 & $\mathrm{Z}$ & $\begin{array}{l}\text { Carelessness }>\text { Annotation to student. Notification both in/appropriate (no } \\
\text { understanding of plagiarised references) }\end{array}$ \\
\hline 15 & 2010 & $\mathrm{Y}$ & A little disregard. No sanctions \\
\hline 16 & 2017 & $\mathrm{Y}$ & $\begin{array}{l}\text { Violation of good scientific practice found, "more disregard for responsible } \\
\text { conduct of research than purposeful deception". (TENK) }\end{array}$ \\
\hline 17 & 2014 & $\mathrm{X}$ & Re-evaluated, grade lowered. Thesis marked with plagiarism statement \\
\hline 18 & 2017 & 0 & $\begin{array}{l}\text { Rector answered by email after reminder. No investigation, not considered a } \\
\text { violation }\end{array}$ \\
\hline 19 & 2014 & $\mathrm{Z}$ & $\begin{array}{l}\text { No violation, no sanctions. Student very upset. (No understanding of plagiarised } \\
\text { references.) (TENK) }\end{array}$ \\
\hline 20 & 2012 & 0 & $\begin{array}{l}\text { No inquiry. According to decision inquiry took place in } 2013 \text { (not attached), } \\
\text { violation not found. Removed from Theseus }\end{array}$ \\
\hline 21 & 2017 & $\mathrm{X}$ & $\begin{array}{l}39 \% \text { similarity with a previous thesis. Violation }>\text { re-evaluation. Thesis marked } \\
\text { with plagiarism statement. Removed from Theseus }\end{array}$ \\
\hline 22 & 2017 & $\mathrm{Z}$ & $\begin{array}{l}\text { Preliminary enquirer did not find a violation (no understanding of plagiarised } \\
\text { references). (TENK) }\end{array}$ \\
\hline 23 & 2018 & $\mathrm{Y}$ & $\begin{array}{l}\text { Preliminary inquiry: reason to doubt disregard of good scientific practise. } \\
\text { Investigation proper started }\end{array}$ \\
\hline 24 & 2012 & $0 / \mathrm{X}$ & $\begin{array}{l}\text { Email from the rector after reminder. No inquiry. Plagiarism was already found } \\
\text { before acceptance }\end{array}$ \\
\hline 25 & 2012 & 0 & No inquiry due to time lapse. (TENK) \\
\hline 26 & 2017 & XY & Re-evaluation \\
\hline 27 & 2015 & 0 & $\begin{array}{l}\text { No inquiry, case too old. Only the person whose text has been plagiarised can } \\
\text { make a notification. (TENK) }\end{array}$ \\
\hline 28 & 2015 & 0 & $\begin{array}{l}\text { No inquiry, case too old. Only the person whose text has been plagiarised can } \\
\text { make a notification. (TENK) }\end{array}$ \\
\hline 29 & 2012 & 0 & $\begin{array}{l}\text { No inquiry, case too old. Only the person whose text has been plagiarised can } \\
\text { make a notification. (TENK) }\end{array}$ \\
\hline
\end{tabular}

$\mathrm{X}=$ plagiarism stated, $\mathrm{Y}=$ disregard stated, $\mathrm{Z}=$ no violation found, $\mathrm{V}=$ violation, not specified, $0=$ no investigation, $(\mathrm{TENK})=$ Statement requested from TENK

Publisher's Note Springer Nature remains neutral with regard to jurisdictional claims in published maps and institutional affiliations. 